\title{
Originales
}

\section{Cirugía cardíaca en pacientes de edad avanzada}

\author{
S. JUSTa, T.M. TOMASA ${ }^{a}$, P. MARCOS ${ }^{a}$, L. BORDEJÉa ${ }^{a}$ P. TORRABADELLA ${ }^{a}$, H.P. MOLTÓa, \\ J.A. MORENO ${ }^{a}$ Y M.A. CASTRO \\ aServicio de Medicina Intensiva. ' Unidad de Cirugía Cardíaca. Hospital Universitari Germans Trias i Pujol. \\ Badalona. Barcelona. España.
}

Objetivo. Comparar la morbilidad y la mortalidad de los pacientes de cirugía cardíaca de acuerdo a la edad inferior o superior a 75 años.

Diseño. Estudio descriptivo retrospectivo.

Pacientes. Dos mil cuatrocientos setenta pacientes consecutivos ingresados en el postoperatorio inmediato tras cirugía cardíaca en nuestra Unidad de Medicina Intensiva entre noviembre de 2000 y diciembre de 2005. De ellos, 1.983 eran menores de 75 años y 497 mayores de 75 años. Se han incluido todos los pacientes con cirugía, tanto programada como urgente y emergente.

Principales variables de interés. Factores de riesgo cardiovascular (diabetes mellitus, hipertensión arterial y dislipidemia), European System for Cardiac Operative Risk Evaluation (EuroSCORE) y EuroSCORE logístico, estancia, mortalidad, complicaciones durante la estancia en la Unidad de Cuidados Intensivos (UCI).

Resultados. La mortalidad hospitalaria de los pacientes mayores de 74 años fue significativamente superior $(9,2 \%$ frente a $4,3 \%, p<0,05)$. La morbilidad de los pacientes mayores de 74 también fue superior (EuroSCORE 8,2 \pm 2,7 frente a 4,9 $\pm 3,3, p<0,001$ ). Tanto la estancia en la UCI como la estancia hospitalaria fueron significativamente superiores en los pacientes mayores de 74 años.

Conclusiones. En nuestra serie tanto la morbilidad como la mortalidad de los mayores de 74 es superior, lo que conlleva peores resultados en la cirugía cardíaca de estos pacientes.

Correspondencia: Dra. S. Just.

Servicio de Medicina Intensiva.

Unidad de postoperatorio de cirugía cardíaca

Hospital Germans Trias i Pujol

Carretera de Canyet, s/n.

08917 Badalona. Barcelona. España.

Correo electrónico: msjust@gmail.com

Manuscrito aceptado el 17-VII-2007.
PALABRAS CLAVE: cirugía cardíaca, esternotomía, circulación extracorpórea, mortalidad, cuidados postoperatorios, ancianos.

\section{CARDIAC SURGERY IN ELDERLY PATIENTS}

Objective. To compare morbidity and mortality of cardiac surgery patients according to age below or above 75 years.

Design. Descriptive retrospective study.

Patients. A total of 2,470 consecutive patients admitted to our Intensive Medicine Unit between November 2000 and December 2005 who were in the immediate postoperative period after cardiac surgery. Of these patients, 1,983 were younger than 75 years and 497 were older than 75 years.

Main variables of interest. Cardiovascular risk factors (diabetes mellitus, arterial hypertension and dyslipidemia), EuroSCORE (European System for Cardiac Operative Risk Evaluation) and logistic EuroSCORE, length of stay, mortality, complications during Intensive Care Unit (ICU) stay.

Results. In-hospital mortality of patients older than 74 years was significantly higher $(9.2 \%$ versus $4.2 \%, p<0.05)$. The morbidity of patients over 74 years of age was also significantly higher (EuroSCORE $8.2 \pm 2.7$ versus $4.9 \pm 3.3, p<0.001$ ). Both ICU stay and hospital stay were significantly higher in those over 74 years of age.

Conclusions. In our series both morbidity and mortality were higher in those older than 74 years of age group, which entails worse results in cardiac surgery of these patients.

KEY WORDS: cardiac surgery, sternotomy, cardiopulmonary bypass, mortality, postoperative care, elderly.

\section{INTRODUCCIÓN}

El envejecimiento progresivo de la población en los países occidentales en las últimas décadas ha pro- 
ducido numerosos cambios sociales que trascienden muchos campos, como es el ámbito sanitario. Está claro que el aumento de la esperanza de vida es paralelo a una mejoría socioeconómica, y en los últimos años España ha llegado a ponerse a la cabeza de los países con mayor esperanza de vida. El porcentaje de personas mayores de 65 años es de aproximadamente el $17 \%$. La esperanza de vida en las mujeres se sitúa en 82 años y casi en 76 en los hombres ${ }^{1}$. Lo que antiguamente se denominaba tercera edad ha sido ahora desglosada, hablándose de cuarta edad a partir de los 80 años $^{2}$. Todo ello repercute en gran manera en nuestros hospitales y, dentro de ellos, en las Unidades de Cuidados Intensivos (UCI) ${ }^{3,4}$.

En los inicios de las UCI, uno de los criterios para decidir el ingreso podía ser la edad del paciente. En aquellos tiempos el número de camas de UCI era menor que en la actualidad. En la mayoría de grandes hospitales representaba un 5\% del total de camas hospitalarias, y en muchos centros ni siquiera existía este servicio. Es lógico pensar que el criterio de ingreso debía ser más estricto. En general, un paciente podía considerarse no tributario de UCI con edad superior a 75 años, aunque en todo caso primara el sentido común a la hora de ingresar a un paciente, y se valorara el beneficio de los procedimientos ${ }^{5}$.

Actualmente nos encontramos con pacientes ancianos que tienen buena calidad de vida relacionada con la salud, concepto muy en boga en los últimos $a_{n}{ }^{6,7}$. Nadie podría negar asistencia plena a una persona que está satisfecha con su vida y su estado de salud física y mental ${ }^{8,9}$. En la mayoría de casos, aunque el anciano sea portador de enfermedad crónica como la diabetes mellitus, la hipertensión arterial o la cardiopatía, la asistencia en los centros hospitalarios y la optimización en la Atención Primaria ha mejorado su control $^{10}$. Existen además en la actualidad medios de tratamiento más eficaces, menos agresivos y, en consecuencia, con menos efectos secundarios que pueden, además de tratar la enfermedad, mejorar la calidad de vida del paciente ${ }^{11}$.

En el medio hospitalario atendemos, entre otros, tres tipos diferentes de pacientes ancianos: a) ancianos previamente sanos que presentan enfermedad aguda que puede ser traumática, médica o quirúrgica; b) ancianos con enfermedad conocida no invalidante y que presentan una descompensación o enfermedad derivada de esta, y c) ancianos con patología que les hace dependientes por invalidez mental y/o física, mala calidad de vida relacionada con la salud y/o patologías de tipo terminal.

En general, los pacientes que se someten a cirugía cardíaca pertenecen a los grupos a y b anteriormente mencionados, pero en algunos casos, por varios motivos, son remitidos al hospital pacientes del grupo c. En este último caso nos encontramos con dificultades a la hora de tomar decisiones, en cuanto al tipo de tratamiento inmediato y en cuanto al seguimiento posterior.

Cada día es mayor el número de ancianos sometidos a cirugía de alto riesgo, al poderles ofrecer con cierta garantía una probabilidad de mejorar su calidad de vida ${ }^{12}$. La optimización de las técnicas, los materiales y medios, hace posible minimizar los riesgos ${ }^{13}$. Sin embargo, suele tratarse de pacientes que presentan comorbilidades por patología asociada y que por tanto son susceptibles de sufrir más complicaciones ${ }^{14-17}$.

Este aumento progresivo de pacientes mayores de 75 años que se someten a cirugía cardíaca en nuestro hospital nos impulsó a iniciar un estudio que nos ayudara a comparar el seguimiento postoperatorio en estos enfermos con los menores de 75 años.

\section{PACIENTES Y MÉTODOS}

En nuestro hospital se inició la cirugía cardíaca en noviembre del año $2000^{18}$. Se intervienen más de 500 pacientes al año. El servicio de UCI dispone de una unidad dotada de 8 camas exclusivamente para control postoperatorio de este tipo de cirugía.

Este estudio se ha realizado con los pacientes intervenidos entre noviembre de 2000 y diciembre de 2005. La información se ha recogido de forma retrospectiva para los pacientes ingresados hasta diciembre de 2004, y de manera prospectiva durante el 2005. Se han incluido todos los pacientes con cirugía, ya fuera programada, urgente o emergente. El análisis se ha realizado separando la población de estudio en dos grupos: edad $<75$ años y edad $\geq 75$ años.

Se han registrado datos de filiación (edad y sexo), factores de riesgo (diabetes mellitus, hipertensión arterial y dislipidemia), European System for Cardiac Operative Risk Evaluation (EuroSCORE) ${ }^{19}$ y EuroSCORE logístico ${ }^{20}$, estancia en la UCI, y mortalidad en la UCI y en el hospital.

Los tipos de cirugía se han clasificado del siguiente modo: cirugía de revascularización coronaria, valvular en general, combinada y miscelánea. Hemos recogido además el porcentaje de casos con cirugía de válvula aórtica única, por ser un procedimiento más habitual en pacientes de la cuarta edad, y con una entidad y evolución propias.

Otras variables sobre las que se ha obtenido información son las siguientes: utilización o no de bomba de circulación extracorpórea (CEC), tiempo de CEC y de clampaje aórtico, horas de ventilación mecánica (VM), realización o no de traqueostomía percutánea, de contrapulsación aórtica, de técnicas continuas de reemplazo renal o hemodiálisis, de cardioversión eléctrica (CE) y necesidad de marcapasos definitivo.

En cuanto a las complicaciones, se ha recogido información sobre las puramente derivadas de la cirugía, como pueden ser reintervenciones por sangrado excesivo, taponamiento pericárdico y mediastinitis, y otras frecuentemente observadas en pacientes de cirugía cardíaca como insuficiencia renal, fibrilación auricular, infecciones, infarto de miocardio (IAM) perioperatorio, prolongación de VM más de 96 horas, accidente cerebrovascular agudo (ACVA), isquemia intestinal, bloqueo auriculoventricular completo y parada cardíaca. Como insuficiencia renal se consideraron los casos de insuficiencia renal aguda (según escala RIFLE) ${ }^{21}$ y crónica agudizada. La fibrilación 
TABLA 1. Características de los pacientes incluidos en el estudio

\begin{tabular}{|l|c|c|c|}
\hline & $<75$ años $(\mathrm{n}=1.983)$ & $\geq 75$ años $(\mathrm{n}=497)$ & $\mathrm{p}$ \\
\hline Edad (años) & $61,1 \pm 10,5$ & $77,6 \pm 2,4$ & $<0,001$ \\
Varones & $1.384(69,7 \%)$ & $263(52,9 \%)$ & $<0,001$ \\
HTA & $1.063(53,7 \%)$ & $322(64,9 \%)$ & 0,001 \\
Diabetes mellitus & $628(31,7 \%)$ & $146(29,3 \%)$ & 0,2 \\
Dislipidemia & $1.019(51,3 \%)$ & $216(43,4 \%)$ & $<0,001$ \\
EuroSCORE (puntos) & $4,9 \pm 3,3$ & $8,2 \pm 2,7$ & $<0,001$ \\
EuroSCORE logístico (puntos) & $6,5 \pm 10,1$ & $12,7 \pm 13,1$ & 0,003 \\
Estancia en UCI (días) & $4,2 \pm 6,8$ & $5,4 \pm 8,5$ & 0,004 \\
Estancia hospitalaria (días) & $12,8 \pm 11,9$ & $15,5 \pm 13,1$ & $<0,05$ \\
Mortalidad hospitalaria & $86(4,3 \%)$ & $46(9,2 \%)$ & \\
\hline
\end{tabular}

Los valores se presentan como media \pm desviación estándar. HTA: hipertensión arterial; UCI: Unidad de Cuidados Intensivos.

auricular (FA) se consideró durante la estancia hospitalaria, incluyendo días en la UCI y en planta, y aquellas que precisaron o no CE. No se incluyeron los pacientes con FA crónica. Las infecciones se refieren a pacientes con sepsis y hemocultivos positivos. Se consideró IAM perioperatorio si se presentaba elevación significativa del segmento ST de nueva aparición y cifras elevadas de troponina I. En los casos de cirugía coronaria, se consideró significativa la troponina I $>10 \mathrm{ng} / \mathrm{ml}$, mientras que en pacientes valvulares si era $>40 \mathrm{ng} / \mathrm{ml}$. En cualquier caso, se valoraron los cambios eléctricos y hemodinámicos significativos y se realizó valoración mediante ecocardiografía urgente. En cuanto a los eventos neurológicos, destacamos los que presentaron ACVA con focalidad neurológica y efecto transitorio o permanente. No se han incluido los casos de encefalopatías transitorias como desorientación, agitación, alteraciones de la memoria y delirios. Los pacientes con alta sospecha de isquemia intestinal fueron en todos los casos sometidos a laparostomía que confirmó el diagnóstico. En cuanto al bloqueo auriculoventricular, destacamos los casos de bloqueo completo, contabilizando los transitorios y los permanentes que precisaron marcapasos endocavitario.

\section{Análisis estadístico}

Los valores están expresados como media \pm desviación estándar o como porcentajes. Las comparaciones entre variables continuas han sido realizadas mediante la prueba de Student-Newman-Keuls. Las comparaciones entre proporciones se han realizado con la prueba de la Chi-cuadrado. Un valor de p menor de 0,05 se ha considerado estadísticamente significativo.

\section{RESULTADOS}

Durante 5 años han ingresado un total de 2.480 pacientes, de los cuales un $20 \%$ son mayores de 74 años. De estos, un $18 \%$ son mayores de 80 años. Las características de los pacientes incluidos en el estudio de acuerdo a la edad se presentan en la tabla 1. Los pacientes mayores de 74 años presentaron un porcentaje significativamente mayor de comorbilidad, una puntuación más alta en las escalas de riesgo quirúrgico, una estancia en la UCI y en el hospital más prolongada, y el doble de mortalidad hospitalaria que los pacientes menores de 75 años.

Los tipos de cirugía realizados se presentan en la tabla 2, donde se puede observar que la cirugía coronaria es más frecuente en los menores de 75 años, mientras que la cirugía valvular es más frecuente en los mayores de 74 años.

En la tabla 3 se muestran los procedimientos realizados durante la estancia hospitalaria. El tiempo de VM y la utilización de marcapasos definitivo, CE, hemofiltración continua y traqueostomía percutánea fueron significativamente mayores en el grupo de pacientes de más de 74 años.

Las complicaciones aparecidas durante el ingreso se muestran en la tabla 4. Los pacientes mayores de 74 años tuvieron una frecuencia significativamente mayor de insuficiencia renal, FA, bloqueo auriculoventricular, sepsis con bacteriemia y necesidad de VM prolongada.

\section{DISCUSIÓN}

Actualmente un 5\% de la población mundial es mayor de 80 años. En países desarrollados la previsión es que en el 2020 exista un 15\% de mayores de 80 años y en el 2050 un 25\%. Este progresivo enve-

TABLA 2. Tipos de cirugía realizada en los pacientes incluidos en el estudio

\begin{tabular}{|c|c|c|c|}
\hline & $<75$ años $(\mathrm{n}=1.983)$ & $\geq 75$ años $(\mathrm{n}=497)$ & $\mathrm{p}$ \\
\hline Cirugía coronaria & $966(48,7 \%)$ & $146(29,4 \%)$ & $<0,001$ \\
\hline Cirugía valvular & $896(45,2 \%)$ & $314(63,3 \%)$ & $<0,001$ \\
\hline Cirugía combinada & $160(8,1 \%)$ & $90(18,1 \%)$ & $<0,001$ \\
\hline Cirugía de válvula aórtica & $254(12,8 \%)$ & $138(27,8 \%)$ & $<0,001$ \\
\hline Miscelánea & $105(5,3 \%)$ & $24(4,8 \%)$ & 0,67 \\
\hline
\end{tabular}


TABLA 3. Procedimientos realizados durante la estancia hospitalaria en los pacientes incluidos en el estudio

\begin{tabular}{|c|c|c|c|}
\hline & $<75$ años $(\mathrm{n}=1.983)$ & $\geq 75$ años $(\mathrm{n}=497)$ & $\mathrm{p}$ \\
\hline Cirugía extracorpórea & $1.830(92,3 \%)$ & $454(91,3 \%)$ & 0,48 \\
\hline Tiempo de cirugía extracorpórea (minutos) & $106,1 \pm 42,2$ & $105,3 \pm 40,9$ & 0,7 \\
\hline Tiempo de clampaje (minutos) & $70,6 \pm 32,8$ & $73,2 \pm 30,8$ & 0,13 \\
\hline Ventilación mecánica (horas) & $29,8 \pm 123,4$ & $44,4 \pm 152,7$ & 0,02 \\
\hline Marcapasos definitivo & $28(1,4 \%)$ & $19(3,8 \%)$ & 0,001 \\
\hline Traqueostomía percutánea & $64(3,2 \%)$ & $33(6,6 \%)$ & 0,001 \\
\hline Balón de contrapulsación aórtico & $22(1,6 \%)$ & $9(1,8 \%)$ & 0,2 \\
\hline Hemofiltración continua & $72(3,6 \%)$ & $30(6,8 \%)$ & 0,01 \\
\hline Cardioversión eléctrica & $52(2,6 \%)$ & $23(4,6 \%)$ & 0,02 \\
\hline
\end{tabular}

jecimiento de la población en los países occidentales está conduciendo a un mayor número de pacientes ancianos sometidos a cirugía cardíaca. En algunos países la cirugía cardíaca en mayores de 80 años alcanza ya entre el 4 y el $12,3 \%$ del total ${ }^{22}$. Parece que el envejecimiento poblacional incrementará la cirugía de revascularización coronaria, de valvulopatía aórtica degenerativa, de regurgitación mitral, de insuficiencia cardíaca, y los procedimientos combinados valvulares y de revascularización ${ }^{23}$. Sin embargo, a pesar de que el perfil del paciente de cirugía cardíaca es más anciano y con procedimientos quirúrgicos más complicados, los resultados postoperatorios son similares en los últimos años a los conseguidos en pacientes con menor edad. Actualmente se cree que el mayor riesgo operatorio de los pacientes debido al aumento de la edad no tendría necesariamente que empeorar los resultados ${ }^{24}$.

Los datos de las series reportadas de pacientes ancianos son discordantes. Carrascal et $\mathrm{al}^{25}$ analizaron una serie de 120 pacientes de 80 años de edad o más, comparándolos con un grupo de 2.425 pacientes menores de 80 años operados en el mismo período. La mortalidad hospitalaria del grupo de 80 años o más fue del $15,8 \%$, casi el doble que la del grupo de menores de 80 años, que fue del $8,1 \%$. A pesar de una mayor comorbilidad preoperatoria y de una peor clase funcional, la mortalidad en esta serie de octogenarios fue aceptable y la calidad de vida postoperatoria muy satisfactoria.

Bridges et $\mathrm{al}^{22}$ usaron la base de datos de la Society of Thoracic Surgeons para revisar retrospectivamente 662.033 pacientes ( 5 pacientes de más de 100 años;
1.092 pacientes de 90 a 99 años; 59.576 pacientes de 80 a 89 años, y 621.360 pacientes de 50 a 79 años) que fueron sometidos a procedimientos quirúrgicos cardíacos entre 1997 y 2000. Se desarrolló un modelo multivariado de regresión logística para examinar los factores asociados de manera independiente con la mortalidad operatoria en pacientes de más de 90 años de edad. Aproximadamente el 57\% de los nonagenarios y centenarios no tenía ninguno de los cuatro principales factores de riesgo (cirugía emergente o de salvamento, balón de contrapulsación aórtico, insuficiencia renal, enfermedad vascular periférica o cerebral) y su mortalidad operatoria fue sólo del 7,2\%. La conclusión de estos autores es que la mortalidad operatoria y la frecuencia de complicaciones asociadas son mayores para los nonagenarios y los centenarios, pero que con una selección cuidadosa de los pacientes la mayoría de ellos tienen un menor riesgo de mortalidad asociada al bypass coronario arterial por injerto, que se acerca al de los pacientes jóvenes.

Diversos autores no han hallado que la edad sea un predictor de mortalidad. Gersbach et $\mathrm{al}^{26}$ analizaron los factores que podían influir en el pronóstico y, por lo tanto, sugerir limitación del esfuerzo terapéutico en 1.859 pacientes postoperados de cirugía cardíaca que requirieron 5 días o más de ingreso en la UCI, y no pudieron identificar ninguno, ni siquiera la edad. Utilizando un análisis de regresión logística multivariado de 19 variables de mala evolución, solamente pudieron identificar la diálisis como factor reductor de la supervivencia a largo plazo, y el déficit neurológico mantenido como predictor de mal pronóstico funcional.

TABLA 4. Complicaciones aparecidas durante el ingreso hospitalario en los pacientes incluidos en el estudio

\begin{tabular}{|c|c|c|c|}
\hline & $<75$ años $(\mathrm{n}=1.983)$ & $\geq 75$ años $(\mathrm{n}=497)$ & $\mathrm{p}$ \\
\hline Insuficiencia renal & $278(13,9 \%)$ & $141(28,2 \%)$ & $<0,001$ \\
\hline Ventilación mecánica $>96$ horas & $114(5,7 \%)$ & $50(10,1 \%)$ & 0,001 \\
\hline Accidente cerebrovascular & $40(2,0 \%)$ & $15(3,0 \%)$ & 0,17 \\
\hline Fibrilación auricular & $494(24,7 \%)$ & $189(37,8 \%)$ & $<0,001$ \\
\hline Bloqueo auriculoventricular & $72(3,6 \%)$ & $39(7,9 \%)$ & $<0,001$ \\
\hline Infarto de miocardio & $60(3,0 \%)$ & $15(3,0 \%)$ & 0,99 \\
\hline Reintervención por sangrado & $48(2,4 \%)$ & $19(3,8 \%)$ & 0,08 \\
\hline Taponamiento pericárdico & $22(1,1 \%)$ & $11(2,2 \%)$ & 0,05 \\
\hline Hemocultivos positivos & $48(2,4 \%)$ & $45(9,0 \%)$ & $<0,001$ \\
\hline Mediastinitis & $10(0,5 \%)$ & $5(1,0 \%)$ & 0,19 \\
\hline
\end{tabular}


Vohra et al ${ }^{27}$ estudiaron un grupo de 8.314 pacientes consecutivos de cirugía cardíaca con CEC durante 7 años para analizar los factores de reingreso en UCI. Un 2,3\% necesitaron volver a la UCI. En el análisis multivariado los factores que mejor predijeron el reingreso fueron la aparición durante el primer ingreso de complicaciones respiratorias, estado de bajo gasto, arritmias, insuficiencia renal que requiriera hemofiltración, y reexploración por hemorragia. Bapat et $\mathrm{al}^{28}$ estudiaron a 804 pacientes postoperados de cirugía cardíaca durante un año consecutivo y analizaron la supervivencia y calidad de vida en los 89 que habían tenido una estancia prolongada ( $\geq 5$ días). Los predictores independientes de mortalidad fueron: el soporte cardíaco preoperatorio (balón de contrapulsación), una fracción de eyección baja, una puntuación más alta en el score Parsonnet y EuroSCORE, las complicaciones pulmonares, la insuficiencia renal que requiriera hemofiltración, las complicaciones del sistema nervioso central y el fallo de tres o más órganos o sistemas. Aunque en el EuroSCORE se penaliza la edad, esta no aparecía como factor independiente de mortalidad.

En cambio, Scott et al $^{29}$, observando su serie de 155 octogenarios y 1.591 pacientes menores de 80 años, encontraron que los octogenarios sometidos a cirugía con CEC requerían una mayor utilización de recursos, y tenían una morbilidad significativamente más alta, con aumento de la incidencia de fallo renal agudo y complicaciones neurológicas, así como mayor mortalidad a los 30 días. Una edad de 80 años o superior era un factor predictivo independiente de mayor utilización de recursos, morbilidad postoperatoria y mortalidad.

Los distintos autores que comunican series de pacientes ancianos difieren en la valoración de los resultados de la cirugía en mayores de 80 años. Por una parte, autores como Carrascal et $\mathrm{al}^{25} \mathrm{o}$ Scott et $\mathrm{al}^{29}$, observan una mayor mortalidad en los mayores de 80; otros en cambio, como Gersbach et $\mathrm{al}^{26}$ y Vohra et $\mathrm{al}^{27}$, no hallan una mortalidad superior relacionada con la edad. Muy paradigmático de estas valoraciones es el análisis de Bridges et $\mathrm{al}^{22}$, que encuentran que, con una selección cuidadosa de los pacientes, la edad no tendría que suponer una mayor mortalidad. Los distintos autores están de acuerdo en que la morbilidad de estos pacientes es más elevada que la de los más jóvenes.

En nuestra serie observamos que la morbilidad, reflejada por el EuroSCORE de los pacientes mayores de 74 es superior (tabla 1). Analizando las variables de evolución, tanto la estancia en UCI como la estancia hospitalaria y la mortalidad hospitalaria son significativamente más elevadas en los mayores de 74 años. La diferencia de mortalidad es apreciable, de $4,3 \%$ en los menores de 75 a 9,2\% en los mayores de 74. Estas diferencias no hacen más que constatar la mayor comorbilidad de nuestros pacientes mayores y su mayor riesgo quirúrgico, tal como muestra el EuroSCORE. Nuestros pacientes de más edad presentan claramente mayor morbilidad y mayor mortalidad.
Como limitación de nuestro estudio está la falta de un análisis de calidad de vida de estos pacientes tanto previo como posterior a la intervención. Nuestra impresión es que aunque son pacientes ya limitados antes de la intervención, estas limitaciones son aún más importantes posteriormente. Sería importante valorar esta calidad de vida postoperatoria, ya que es frecuente constatar que los pacientes presentan un deterioro marcado respecto al que tenían antes de la intervención. Aspectos como la dependencia, la falta de autonomía para las actividades de la vida diaria, la limitación funcional, el dolor o la depresión son tan importantes como la clase funcional. En este estudio no se ha realizado un seguimiento de estos pacientes fuera del hospital para estimar las condiciones de vida. Probablemente la calidad de vida es inferior a la que tendrían si hubieran seguido tratamiento médico.

La tendencia a aumentar las listas de espera, la ampliación de indicaciones de cirugía, el empleo de cirugía como recurso de emergencia en pacientes que antes se hubieran desestimado y como terapia de rescate de otros procedimientos médicos o mínimamente invasivos, puede haber contribuido mucho a estos resultados. Evidentemente, parece que haya una tendencia a considerar la cirugía cardíaca del paciente anciano como una solución mágica a los problemas que no se pueden resolver de otra forma, cuando en realidad muchas veces es una caja de Pandora que al abrir nos puede mostrar una serie de desagradables complicaciones que antes de la intervención no podían sospecharse y que parecía que no pudieran llegar a ocurrir nunca. La conclusión que podemos extraer de estos datos es que no se debe infravalorar el riesgo quirúrgico de estos pacientes, con un desconocimiento de los resultados de esta cirugía, fiándonos en unas series muy optimistas, que posiblemente reflejan más algún tipo de selección que no la realidad de nuestras listas de espera.

\section{Declaración de conflicto de intereses}

Los autores han declarado no tener ningún conflicto de intereses.

\section{BIBLIOGRAFÍA}

1. López-Messa JB. Envejecimiento y medicina intensiva. Med Intensiva. 2005;29:469-74.

2. Laverdad.es Alicante-PROVINCIA-2006 (revista electrónica). Cada vez más gente de la cuarta edad (consultado el 25-12006). Disponible en: http://servicios.laverdad.es/alicante/pg06110/ prensa/noticias/Provincia_Alicante/200601/10/ALI-CUL226.htlm

3. Esteban A, Anzuelo A, Frutos-Vivar F, Alía I, Ely EW, Brochard L, et al. Outcome of older patients receiving mechanical ventilation. Intensive Care Med. 2004;30:639-46.

4. Boumendil A, Aegerter P, Guidet B, and the CUB-Rea Network. Treatment intensity and outcome of patients aged 80 and older in ICU: A multicenter matched-cohort study. J Am Geriatr Soc. 2005;53:88-93.

5. Vosylius S, Sipylaite J, Ivasdkevicius J. Determinants of outcome in elderly patients admitted to the ICU. Age Ageing. 2005; 34:157-62.

6. Dowdy DW, Eid MP, Sedrakyan A, Mendez-Tellez PA, Pronosvost PJ, Herridge MS, et al. Quality of life in adult survivors 
of critical illness: a systematic review of the literatura. Intensive Care Med. 2005;31:611-20.

7. Boumendil A, Maury E, Reinhard I, Luquel L, Offenstad G, Guidet B. Prognosis of patients aged 80 years and over admitted in medical ICU. Intensive Care Med. 2004;30:647-54.

8. Janse A, Gemke R, Uiterwaal C, van der Tweel I, Kimpen JL, Sinnema G. Quality of life: patients and doctors don't always agree: a meta-analysis. J Clin Epidemiol. 2004;57:653-61.

9. Koch CG, Khandwala F, Cywinski JB, Ishwaran H, Estefanous FG, Loop FD, et al. Health-related quality of life after coronary artery bypass grafting: a gender analysis using the Duke Activity Status Index. J Thorac Cardiovasc Surg. 2004;128:284-95.

10. Rellos K, Falagas ME, Vardakas KZ, Sermaides G, Michalopoulos A. Outcome of critically ill oldest-old patients (aged 90 and older) admitted to the intensive care unit. J Am Geriatr Soc. 2006;54:110-4.

11. Chocron S, Tatou E, Schjoth B, Naja G, Clement F, Viel JF, et al. Perceived health status in patients over 70 before and after open-heart operations. Age Ageing. 2000;29:329-34.

12. Bachetta MD, Ko W, Girardi LN, Mack CA, Krieger KH, Isom OW, et al. Outcomes of cardiac surgery in nonagenarians: a 10 years experience. Ann Thorac Surg. 2003;75:1215-20.

13. Juffé A. Cirugía coronaria en la tercera edad. Rev Esp Cardiol. 2001;54:677-8.

14. Hirose H, Amano A, Takahashi A. Coronary artery bypass grafting for octogenarians: experience in a private hospital and review of the literature. Ann Thorac Cardiovasc Surg. 2001;7:28291.

15. Hewitt TD, Santa Maria PL, Alvarez JM. Cardiac surgery in Australian octogenarians:1996-2001. ANZ J Surg. 2003;73: 749-54.

16. Chiappini B, Camurri N, Loforte A, Di Marco L, Di Bartolomeo R, Marinelli G. Outcome after aortic valve replacement in octogenarians. Ann Thorac Surg. 2004;78:85-9.

17. Blackman DJ, Ferguson JD, Sprigings DG, Banning AP. Revascularization for acute coronary syndromes in older people. Age Ageing. 2003;32:129-35.
18. Just S, Tomasa TM, Ricart P, Sánchez S, Moreno JA, Pérez HE, et al. Cirugía cardíaca en pacientes de edad avanzada. Med Intensiva. 2005; 29 Supl 1:31.

19. Hashef SA, Roques F, Michel P, Gaudcheau E, Lemeshow S, Salamon R. European system for cardiac operative risk evaluation (EuroSCORE). Eur J Cardiothorac Surg. 1999;16:9-13.

20. Michel P, Roques F, Nashef SA; EuroSCORE Project Group. Logistic or additive EuroSCORE for high-risk patients? Eur J Cardiothorac Surg. 2003;23:684-7.

21. Kellum JA, Bellomo R, Ronco C. The concept of acute kidney injury and the RIFLE criteria. Contrib Nephrol. 2007;156: $10-6$.

22. Bridges CR, Edwards FH, Peterson ED, Coombs LP, Ferguson TB. Cardiac surgery in nonagenarians and centenarians. J Am Coll Surg. 2003;197:347-57.

23. Turina MR. Future of the heart valve surgery. Eur J Cardiothorac Surg. 2004;26 Suppl 1:8-13.

24. Aggarwal SK, Fox SA, Stitt L, Kiaii B, McKenzie FM, Menkis AH, et al. The new cardiac surgery patient: defying previous expectations. Can J Surg. 2006;49:117-22.

25. Carrascal Y, Di Stefano S, Fulquet E, Echevarría JR, Flórez S, Fiz L. Cirugía cardíaca en octogenarios: situación actual y perspectivas de futuro. Med Clin (Barc). 2006;126:170-2.

26. Gersbach P, Tevaearai H, Revelly JP, Bize P, Chiolero R, von Segesser LK. Are there accurate predictors of long-term vital and functional outcomes in cardiac surgical patients requiring prolonged intensive care? Eur J Cardiothorac Surg. 2006;29:466-72.

27. Vohra HA, Goldsmith IR, Rosin MD, Briffa NP, Patel RL. The predictors and outcome of recidivism in cardiac ICUs. Eur J Cardiothorac Surg. 2005;27:508-11.

28. Bapat V, Allen D, Young C, Roxburgh J, Ibrahim M. Survival and quality of life after cardiac surgery complicated by prolonged intensive care. J Card Surg. 2005;20:212-7.

29. Scott BH, Seifert FC, Grimson R, Glass PS. Octogenarians undergoing coronary artery bypass graft surgery: resource utilization, postoperative mortality, and morbidity. J Cardiothorac Vasc Anesth. 2005;19:583-8. 